\title{
INFLUÊNCIA DO ACESSO À ALIMENTAÇÃO E DA CONDIÇÃO SOCIOECONÔMICANO ESTADO NUTRICIONAL DE ESCOLARES
}

ANDRADE, Kalindi Mariane do Espírito Santo. Nutricionista formada pela Universidade de Ribeirão Preto Unaerp. Rua Lia Bignatti Castelli, 209, Geraldo Correia de Carvalho. CEP 14061-430. Ribeirão Preto-SP.

E-mail:kalindi_ka@hotmail.com.

PEREIRA, Rita de Cássia Garcia. Nutricionista e docente do Curso de Graduação em Nutrição do Centro Universitário de Araraquara - Uniara.

\begin{abstract}
RESUMO
O presente trabalho foi desenvolvido com o objetivo de avaliar a relação entre a condição socioeconômica e o estado nutricional de alunos com idade entre 7 e 10 anos. Foi realizado um estudo transversal com 60 crianças matriculadas em uma escola pública do município de Ribeirão Preto. O estado nutricional foi avaliado por meio de aferições de peso e de estatura; a condição socioeconômica de cada família foi classificada considerando-se a renda per capita; e a situação de segurança e insegurança alimentar foi diagnosticada de acordo com a Escala Brasileira de Medida da Insegurança Alimentar. A maioria das crianças estava eutrófica, porém, foi considerável o número de indivíduos com excesso de peso. Grande parte das famílias possuía renda per capita mensal baixa e quase metade da amostra tinha acesso ao benefício do Programa Bolsa Família. Apenas $25 \%$ das famílias se encontravam em situação de segurança alimentar e a prevalência de insegurança alimentar foi maior entre as famílias com rendimento mensal mais baixo. Os resultados confirmam a hipótese do estudo, indicando associação positiva da condição socioeconômica sobre a situação de segurança alimentar e, consequentemente, no estado nutricional dos indivíduos.
\end{abstract}

Palavras-chave: Condição socioeconômica; Segurança alimentar; Estado nutricional; Escolar.

\begin{abstract}
The present work was developed with the objective to evaluate the relationship between the socio-economic condition and the nutritional state of students ranging from seven to ten years old. We have carriedout a transversal study with 60 children enrolled in a public school in the city of Ribeirão Preto. The nutritional state of the children was evaluated through weight and height measurements; the socio-economic condition of each family was classified considering the per capita income and the security situation and food insecurity was diagnosed according to the Brazilian Scale Measure of Food Insecurity. Most children were eutrophic, but there was a considerable number of individuals who were overweight. Great part of the families had low per capita income and almost half the sample had access to the benefit of the "Bolsa Familia" Program. Only 25\% of the families were in food security situation and the prevalence of food insecurity was higher among families with lower monthly income. The results confirm the hypothesis of the study, indicating a positive association of socioeconomic condition on the food security situation and, consequently, on the nutritional status of individuals.
\end{abstract}

KEYWORDs: Socio-economic condition; Food security; Nutritional state; Elementary school students. 


\section{INTRODUÇÃo}

A fase escolar, do ponto de vista educacional, iniciase aos 7 anos de idade e vai até os 14, sendo caracterizada por uma maior socialização e independência da criança, o que, além do aumento do apetite, permite uma melhor aceitação por alimentos e combinações diferentes e mais elaboradas, e ainda o aumento do exercício físico informal, por meio de brincadeiras e diversas atividades, como, por exemplo, andar de bicicleta ou de patins, o que permitirá um maior gasto de energia ao longo do dia - embora seja nessa faixa etária que se dá início ao comportamento sedentário, devido ao ingresso em atividades que não despendem muita caloria, como é o caso da televisão, computador e videogame (VITOLO, 2008).

De acordo com Burlandy e Anjos (2007), o período da idade escolar compreende desde os 7 até os 14 anos de idade; porém, é entre 7 e 10 anos que os dados antropométricos, independentemente de outros parâmetros, são capazes de fornecer informações suficientes para uma avaliação adequada quanto ao estado nutricional, pois os fatores ambientais são ainda considerados predominantes; a partir dos 10 anos de idade, ocorrem diversas modificações entre o indivíduo e as populações que podem interferir no processo de desenvolvimento da criança.

Uma alimentação saudável depende da ingestão de alimentos em quantidade suficiente para cobrir as necessidades energéticas do organismo, completa em sua composição para fornecer ao organismo todos os nutrientes que o integram e que são importantes para o seu bom funcionamento, mas que guardem uma relação de proporção entre si e que seja adequada ao momento biológico da vida do indivíduo ou a alguma enfermidade, respeitando seus hábitos individuais e sua situação econômica e social (TIRAPEGUI; MENDES, 2006).

Segundo Phillippi (2000), as consequências de inadequações nutricionais em populações são detectadas quando uma alimentação errônea é praticada por um longo período de tempo e só então sinais e/ou sintomas de patologias nutricionais são observados; daí a importância dos indicadores precoces do risco dessas patologias, baseados no comportamento alimentar, por meio dos quais é possível orientar os indivíduos e formular programas específicos de nutrição e saúde pública acerca da alimentação saudável.

O estado nutricional é uma condição em que a ingestão e utilização de nutrientes interferem diretamente na saúde e na composição corporal do indivíduo. É medido por meio de indicadores de avaliação nutricional, combase em critérios estatísticos, a partir de uma população de referência considerada saudável (WHO, 1976 apud FERREIRA, 2000, p. 25). De acordo com Ferreira (2000), quando esses indicadores estão acima dos limites de normalidade, há um favorecimento para o estabelecimento de problemas relacionados à alimentação excessiva, tais como: obesidade, diabetes mellitus tipo II, aterosclerose, entre outros; por outro lado, quando a ingestão alimentar não é suficiente ou os nutrientes são pouco aproveitados, ocorre o aparecimento dos agravos carenciais, cujo grande exemplo é a desnutrição energético-proteica, dentre outros problemas.

O nível socioeconômico de uma população está diretamente relacionado ao estado de nutrição desta; a renda per capita, por exemplo, é um fator significativo para aquisição de alimentos em quantidade suficiente para atender às necessidades básicas do indivíduo e da família (LIMA; GOUVEIA, 1999); ou seja, "a ingestão de alimentos depende da despesa com alimentos, que é influenciada pela renda" (BEGHIN, 1999, p.179).

Um estudo realizado por Monteiro et al. (2009) atribui cerca de dois terços do declínio de metade da prevalência de desnutrição às melhorias quanto à escolaridade das mães, ao forte aumento do poder aquisitivo das famílias - principalmente dos mais pobres -, à forte expansão do acesso aos serviços públicos essenciais e às melhores condições de saneamento.

Conforme definição da II Conferência Nacional de Segurança Alimentar e Nutricional - Consea, ocorrida no ano de 2004, a segurança alimentar e nutricional é a realização do direito de todos ao acesso regular e permanente a alimentos de qualidade, em 
quantidade suficiente, sem comprometer o acesso a outras necessidades essenciais, tendo como base práticas alimentares promotoras de saúde, que respeitem a diversidade cultural e que sejam social, econômica e ambientalmente sustentáveis (BURLANDY, 2007, p. 1443).

Inserido no contexto de Segurança Alimentar e Nutricional está o Programa Nacional de Alimentação Escolar-PNAE, que tem por objetivo contribuir para o crescimento e o desenvolvimento biopsicossocial, a aprendizagem, o rendimento escolar e a formação de práticas alimentares saudáveis dos alunos, por meio de ações de educação alimentar e nutricional e da oferta de refeições que cubram as suas necessidades nutricionais durante o período letivo (BRASIL, 2009b, p.3). A alimentação escolar é agora um programa suplementar à educação; já não mais tem um modelo assistencialista, e entende que a alimentação é um direito humano, portanto, a finalidade do programa é oferecer alimentação de boa qualidade a todos os escolares, suprindo pelo menos $15 \%$ de suas necessidades nutricionais diárias, de maneira que contribua para o crescimento e desenvolvimento da criança, favorecendo seu aprendizado e influenciando inclusive na formação de bons hábitos alimentares (DOMENE et al., 2007).

Faz-se necessário, portanto, a avaliação nutricional das crianças considerando a existência de diversos fatores aqui abordados, que interferem no seu crescimento, desenvolvimento e desempenho escolar. A partir do conhecimento do perfil nutricional de uma dada população é que se torna possível uma intervenção efetiva para a melhoria na qualidade de vida das pessoas.

\section{ObJetivo}

O presente trabalho foi desenvolvido com o objetivo de avaliar o acesso à alimentação dos domicílios estudados (situação de segurança e insegurança alimentar) e a relação deste e da condição socioeconômica com o estado nutricional de alunos com idade entre 7 e 10 anos, matriculados em uma escola pública do município de Ribeirão Preto-SP.

\section{Metodologia}

Foi realizado um estudo transversal em uma escola pública do município de Ribeirão Preto, durante o primeiro semestre do ano de 2009, no qual foram incluídos alunos com idade entre 7 e 11 anos incompletos.

A seleção das turmas foi realizada de forma aleatória, o que resultou inicialmente em um total de 364 alunos de ambos os sexos, na faixa etária entre 7 e 10 anos, representando $83,87 \%$ do total de alunos matriculados na escola com a mesma idade $(n=434)$.

$\mathrm{O}$ projeto de pesquisa do presente trabalho foi submetido ao Comitê de Ética em Pesquisa da Universidade de Ribeirão Preto em 12 de março de 2009, pelo qual foi analisado e aprovado sem restrições, tendo parecer positivo em 17 de abril de 2009, registrado sob o Memorando ComÉt/ n. ${ }^{\circ}$ 016/09.

Utilizou-se como padrão de referência as novas curvas de crescimento da Organização Mundial de Saúde - OMS (2007), sendo os dados analisados de acordo com os pontos de corte utilizados pelo Ministério da Saúde - Sisvan (BRASIL, 2008). Para a coleta dos dados antropométricos utilizou-se uma balança portátil digital da marca Plenna, com capacidade para 150 quilogramas e graduação em 100 gramas, e um estadiômetro portátil da marca Sanny com capacidade de $110 \mathrm{~cm}$ a $204 \mathrm{~cm}$ e resolução em milímetros.

Por meio de observação direta foram coletadas informações sobre o tipo de refeição oferecida pela escola, o(s) horário(s) em que era servida e sua composição. A análise foi realizada verificando o cardápio de três dias aleatórios, a fim de identificar adequação do cardápio quanto ao proposto pelo PNAE no que diz respeito a calorias, macro e micronutrientes. A finalidade dessa análise foi identificar a(s) refeição(ões) realizada(s) pela criança na escola, considerando o acesso à alimentação escolar uma das principais formas de obtenção do alimento pela criança. O cardápio da alimentação escolar foi analisado utilizando-se a Tabela de Composição de Alimentos (PHILIPPI, 2002), para se obter a composição quanto aos macro e 
micronutrientes e quanto ao valor calórico oferecido pelas refeições. Como parâmetros para análise da adequação das refeições oferecidas pela Alimentação Escolar, foram utilizados os valores de referências preconizados pelas Resoluções n. 32 e n. ${ }^{\mathbf{o}} 38$ do Fundo Nacional de Desenvolvimento da Educação (BRASIL, 2006; BRASIL, 2009).

Em visita domiciliar foi aplicado um recordatório alimentar habitual para avaliar as refeições realizadas rotineiramente pela criança em casa. Foram registrados todos os alimentos que a criança costuma consumir durante o dia, identificados por refeição. Os alimentos relatados no inquérito dietético foram agrupados de acordo com a classificação estabelecida pela Pirâmide Alimentar Adaptada (PHILIPPI et al., 1999). A avaliação do consumo alimentar foi realizada de acordo com os grupos alimentares: pães, cereais, raízes e tubérculos; hortaliças; frutas; carnes; leite; leguminosas; óleos e gorduras; açúcares e doces; ou seja, os alimentos consumidos relatados por meio do inquérito dietético foram agrupados de acordo com a classificação dos grupos, considerando as porções consumidas, e o consumo comparado às porções estabelecidas pela pirâmide alimentar adaptada a fim de verificar se o consumo se encontrava adequado.

O questionário da Pesquisa Nacional por Amostra de Domicílio - PNAD/IBGE (Brasil, 2004b) foi adaptado, com 43 perguntas quanto à renda familiar $\mathrm{e}$ renda per capita, escolaridade e ocupação do responsável com quem reside a criança, acesso à água tratada, condição de saneamento básico e da moradia e a forma como os alimentos para consumo da criança e de toda a família são obtidos, priorizando o acesso à alimentação escolar e às políticas de transferência de renda. Esse questionário contemplava também as perguntas direcionadas para a identificação da situação de segurança e insegurança alimentar da família, de acordo com a Escala Brasileira de Medida da Insegurança Alimentar - Ebia (BRASIL, 2006), e foi aplicado diretamente à mãe ou responsável pelo menor, em visita domiciliar.
A condição socioeconômica de cada família foi analisada considerando-se principalmente a renda per capita mensal. Para utilização desse critério e análise do seu impacto no estado nutricional das crianças, e também sobre outras variáveis, foi estabelecida a renda per capita mediana ${ }^{1}$. Dessa forma, as famílias estudadas foram divididas em dois grupos: Grupo 1, composto por famílias com renda per capita inferior à renda per capita mediana (RPIM), e o Grupo 2, formado pelas famílias com renda per capita superior à renda per capita mediana (RPSM).

O salário mínimo foi utilizado como critério para a classificação dos indivíduos quanto à renda per capita mensal, que na data da pesquisa equivalia a $\mathrm{R} \$ 465,00$ mensais. Os sujeitos foram distribuídos em cinco faixas salariais, sendo a primeira a renda inferior $\mathrm{a}^{1 / 4}$ do salário mínimo (menor que $\mathrm{R} \$ 116,25$ ); a segunda, entre $1 / 4 \mathrm{e}$ 1/2 salário mínimo (entre R\$ 116,25 e R\$ 232,50); a terceira, de $1 / 2$ a $3 / 4$ do salário mínimo (entre $\mathrm{R} \$ 232,50$ e R \$ 348,75); a quarta, entre 3/4 e 1 salário mínimo (entre $\mathrm{R} \$ 348,75$ e $\mathrm{R} \$ 465,00$ ); e a quinta faixa salarial, a renda per capita maior que 1 salário mínimo (maior que $\mathrm{R} \$ 465,00)$.

A tabulação dos dados foi realizada com a utilização do aplicativo Excel do Microsoft Office 2007, sendo as variáveis qualitativas analisadas por meio de frequência de resposta e as variáveis quantitativas, por meio de média e desvio padrão.

A realização da análise estatística se deu de maneira bastante simples a partir da aplicação do teste quiquadrado, utilizado para comparação entre dois ou mais grupos independentes, que não eram necessariamente do mesmo tamanho. O resultado desse teste foi considerado, para diferença significativa, quando menor que $0,05(\mathrm{p}<0,05)$, e para diferença extremamente significativa, quando menor que 0,001 $(\mathrm{p}<0,001)$.

\section{RESUltadoS E DISCUSSÃo}

A amostra foi composta de 60 crianças, número equivalente a $13,82 \%$ da população total de alunos da

${ }^{1}$ Número central de um determinado conjunto de números (MICROSOFT EXCEL, 2007). 
escola ( $n=434)$, na faixa etária entre 7 e 11 anos incompletos, estudantes dos períodos da manhã e da tarde. Prevaleceu o sexo feminino, com $55 \%$ da amostra. Os alunos tinham idade entre 7 e 10 anos, e a média de idade era de 8,33 $\pm 1,02$ anos.

A prevalência de baixa estatura para idade na amostra foi pequena, visto que apenas $6,67 \%$ das crianças $(n=4)$ apresentaram tal inadequação nutricional do ponto de vista antropométrico. Por meio dos parâmetros de peso para idade e IMC para idade foi possível verificar uma maior prevalência de peso adequado ou eutrófico e IMC adequado ou eutrófico, com $82 \%(n=41)$ e $65 \%(n=39)$, respectivamente. Nenhuma criança foi classificada como baixo peso para a idade; porém, 8,33\% $(\mathrm{n}=5)$ apresentaram baixo IMC para a idade.

Do total de crianças estudadas, $35 \%(n=21)$ apresentaram estado nutricional inadequado - $11,67 \%$ $(n=7)$ e $15 \%(n=9)$ apresentam sobrepeso e obesidade, respectivamente. Ocorreram resultados parecidos em um estudo realizado também no município de Ribeirão Preto-SP com crianças do ensino fundamental; segundo Zancul (2004), emrelação ao IMC para idade, a maioria das crianças apresentou-se eutrófica $(71,6 \%), 21,1 \%$ delas apresentaram excesso de peso, sendo o valor encontrado para sobrepeso de $12,6 \%$ e a obesidade apareceu em $8,6 \% ; 7,1 \%$ das crianças encontravamse com baixo peso.

Do total de pais ou responsáveis entrevistados, $5 \%$ $(n=3)$ não eram alfabetizados, ou seja, não sabiam ler e escrever. Quanto à escolaridade, a grande maioria, $46,67 \%(n=28)$, relatou ter estudado do quarto ano do ensino fundamental até o nono ano do ensino fundamental incompleto.

De acordo com o número de pessoas moradoras no domicílio, a média foi de 5,27 \pm 1,91 pessoas; houve uma maior frequência de quatro pessoas por domicílio, com 36,67\% $(n=22)$, seguida de cinco pessoas, com 26,67\% ( $n=16)$. Em 51,67\% dos domicílios $(n=31)$, apenas uma pessoa contribuía com a renda familiar mensal. Segundo o Instituto Brasileiro de Geografia e Estatísticas - IBGE, a densidade de moradores é mais elevada nos domicílios com menor rendimento, passando de 4,8 nos domicílios cujo rendimento era de até 1/4 de salário mínimo per capita para 2,9 nos domicílios, com mais de três salários mínimos per capita (BRASIL, 2004c).

Dentre as famílias entrevistadas, $15 \%(\mathrm{n}=9)$ residiam em domicílio particular improvisado, ou seja, tinham suas moradias localizadas no interior de favelas, com "barracos" construídos em terrenos da prefeitura-uma dessas casas $(1,67 \%)$ foi construída com madeira aproveitada e as demais, 98,33\% (n=59), tinham a alvenaria como material predominante na construção. O dado reflete o quanto a precariedade das condições de moradia está fortemente associada à insegurança alimentar das famílias, uma vez que o fato de não morar em casa de alvenaria aumenta em quase duas vezes a chance de insegurança alimentar (VIANNA; SEGALL-CORRÊA, 2008).

Quanto às condições de moradia e higiene da população estudada, os itens como esgoto tratado, coleta de lixo, energia elétrica, fogão, geladeira e televisão estavam presentes em todos os domicílios; 98,33\% (n=59) possuíam água encanada; 96,67\% $(\mathrm{n}=58)$ contavam com asfalto; $85 \%(\mathrm{n}=51)$ tinham telefone móvel celular; $51,67 \%(\mathrm{n}=31)$ possuíam telefone fixo convencional; rádio estava presente em $90 \%(n=54)$ das residências estudadas; máquina de lavar roupas, em 56,67\% ( $\mathrm{n}=34)$; microcomputador, em 38,33\% $(n=23)$; e acesso à internet, em 23,33\% $(n=14)$ dos domicílios. Apenas 31,67\% (n=19) dos domicílios possuíam filtro de água, importante equipamento para purificação da água para o consumo das pessoas e questões relacionadas à higiene e saúde.

$\mathrm{O}$ atual governo federal utiliza o salário mínimo como parâmetro para dimensionar a pobreza do país; dessa forma, as pessoas com renda per capita domiciliar de até um quarto do salário mínimo são consideradas muito pobres ou indigentes, e as pessoas com renda de até meio salário mínimo domiciliar per capita, classificadas como pobres (SANTOS; MARTINS; SAWAYA, 2008). Considerando esse parâmetro foi possível perceber que $71,67 \%$ da amostra foi classificada como pobre, com renda per capita inferior a $1 \frac{1}{2}$ salário mínimo $-31,67 \%$ dos 
pesquisados eram muito pobres, com renda per capita inferior a $1 \frac{1}{4}$ do salário mínimo.

A média de renda familiar mensal foi de $\mathrm{R} \$ 938,88$ $\pm 661,28$, o que dá a falsa impressão de que se trata de uma população com uma renda mensal elevada, descartando a ideia de se tratar de uma comunidade carente. Entretanto, é importante levar em consideração o número de pessoas moradoras no mesmo domicílio, cuja média foi de 5,27 $\pm 1,91$, mas que chegou a 13 indivíduos compartilhando o mesmo teto; assume extremos de $\mathrm{R} \$ 4.000,00$ por mês a $\mathrm{R} \$ 65,00$ de renda familiar, sendo este último valor obtido pela família por meio do programa Bolsa Família, a única renda fixa do domicílio. O rendimento familiar per capita é uma importante variável determinante no nível de bem-estar das crianças; de acordo com o IBGE (BRASIL, 2006), cerca de $40 \%$ das crianças com até 14 anos vivem em famílias cujo rendimento não ultrapassa $1 / 2$ salário mínimo mensal per capita.

Segundo a percepção da população quanto à renda, 56,67\% dos entrevistados $(\mathrm{n}=34)$ consideravam que a renda total do domicílio não era suficiente para que os moradores tivessem uma alimentação equilibrada e em quantidade suficiente; $35 \%(n=21)$ consideravam que a renda era suficiente; e $8,33 \%$ consideravam a renda razoavelmente suficiente.

Quanto à participação das famílias em programas de transferência de renda, 53,33\% delas $(n=32)$ não estavam inscritas em nenhum tipo de benefício, ao passo que 46,67\% (n=28) recebiam uma média de $\mathrm{R} \$ 81,58 \pm 34,75$ como complementação de renda pelo Programa Bolsa Família.

A transferência de renda se mostrou mais efetiva, uma vez que grande parte das famílias depende mais do benefício do Programa Bolsa família e poucas recebem doação de cesta básica para a sobrevivência; apenas nove pessoas (15\%) revelaram receber ajuda em alimentos de alguma instituição, de empregador ou de alguma pessoa que não fosse moradora do domicílio, como, por exemplo, os parentes.

Com esse dado é possível perceber a mudança que ocorreu quanto aos programas sociais que eram voltados basicamente para doação de alimentos.
Atualmente, em relação à complementação de renda pelo benefício do Programa Bolsa Família, permite que os beneficiários tenham mais dignidade, uma vez que o benefício é visto como a conquista de uma garantia do seu direito como cidadão, ao contrário do que houve no passado, quando as políticas públicas eram basicamente assistencialistas, oferecidas com irregularidade e na maioria das vezes ineficientes, bastante distantes do propósito de segurança alimentar (TARTAGLIA, 1998).

A renda per capita mediana das famílias observada neste estudo foi de R \$ 150,00 mensais. Dividindo-se as famílias em dois grupos, sendo o Grupo 1 composto por famílias com renda per capita inferior à renda $\mathrm{per}$ capita mediana (RPIM), e o Grupo 2 pelas famílias com renda per capita superior à renda mediana (RPSM), observou-se que 51,67\% das famílias ( $\mathrm{n}=31$ ) pertenciam ao Grupo 1 e 48,33\% (n=29) estavam no segundo grupo, com renda per capita mensal superior a R $\$ 150,00$.

Com base nessa classificação, várias comparações podem ser feitas, a fim de se verificar se há diferenças significativas entre os grupos, que possam comprovar a influência da condição socioeconômica no estado nutricional da população estudada.

Houve diferença entre os grupos quanto ao estado nutricional $(\mathrm{p}<0,05) ; 61,54 \%(\mathrm{n}=24)$ das crianças eutróficas pertenciam a famílias com RPIM e, das 21 crianças com IMC inadequado, tanto para baixo IMC para idade quanto para sobrepeso e obesidade, 14 $(66,67 \%)$ pertenciam ao Grupo de RPSM. Um estudo realizado com escolares do Chile corrobora com esta pesquisa, pois revelou que a elevada prevalência de obesidade se concentrou nos grupos pertencentes às classes socioeconômicas mais baixas; porém, o sobrepeso foi mais predominante na classe média-alta (LIBERONA et al. 2008).

Em se tratando de segurança alimentar, com o presente estudo foi possível verificar que $25 \%$ dos domicílios estudados $(\mathrm{n}=15)$ foram classificados em situação de segurança alimentar (SA); 20\% dos indivíduos $(n=12)$ encontram-se em situação de insegurança alimentar leve - IA Leve, ou seja, havia 
pelo menos a preocupação quanto à possibilidade de faltar alimento no futuro próximo; $43,33 \%$ dos entrevistados $(\mathrm{n}=26)$ foram classificados em situação de insegurança alimentar moderada - IA Moderada, pois já estavam expostos a uma restrição qualitativa na alimentação; e 11,67\% (n=7) estavam em uma condição de deficiência quantitativa de alimentos ou mesmo fome entre adultos e crianças da família, que é a insegurança alimentar grave - IA Grave.

A PNAD realizada no ano de 2004, por meio de uma investigação suplementar sobre a condição domiciliar de segurança alimentar, revela que 34,8\% dos aproximadamente 52 milhões de domicílios particulares estimados se encontravam em situação de insegurança alimentar, correspondendo a cerca de 72 milhões de pessoas vivendo em domicílios com insegurança alimentar leve, moderada ou grave. Para 6,5\% dessa população, a restrição alimentar era tamanha que perto de 14 milhões de pessoas conviveram com a experiência de passar fome, pelo menos uma vez, nos 90 dias que antecederam a data da entrevista. As famílias que foram classificadas como em situação de insegurança alimentar moderada ou grave apresentavam falta de recursos para a aquisição de alimentos e, por conseguinte, limitação de acesso qualitativo aos alimentos (BRASIL, 2006).

A média de renda per capita das famílias em situação de segurança alimentar foi de $\mathrm{R} \$ 298,68$, enquanto as famílias em situação de insegurança alimentar contavam com uma média de $\mathrm{R} \$ 168,63$ por pessoa, por mês. O dado mostra a tendência da insegurança alimentar ser mais presente em famílias de baixa renda, sendo a situação de segurança alimentar diretamente proporcional à renda mensal da família. Entretanto, 6 crianças das 15 pertencentes a famílias classificadas em situação de segurança alimentar (40\%) tinham como renda per capita mensal valores inferiores a R \$232,50 ou 1/2 salário mínimo, sendo classificadas, portanto, como pobres, conforme o atual parâmetro utilizado pelo governo federal para dimensionar a pobreza. A informação compartilha com a constatação de Segall-Corrêa (2007), que afirma existir famílias que estejam situadas abaixo da linha de pobreza e apresentam situação de segurança alimentar, ocorrendo também o oposto.

Houve diferença extremamente significativa $(\mathrm{p}=0,001)$ quanto à situação de segurança e insegurança alimentar das famílias com RPIM e com RPSM, ou seja, ocorreu uma considerável discrepância no que diz respeito ao nível de insegurança alimentar, sendo este inversamente proporcional à renda per capita mensal das famílias estudadas. Na Tabela 1, podem-se verificar as diferenças entre os níveis de IA e a situação de SA em família com renda per capita inferior à mediana $\mathrm{e}$ com renda per capita superior à mediana.

Tabela 1 - Distribuição dos alunos participantes da pesquisa $(n=60)$ de acordo com a situação de segurança e insegurança alimentar do domicílio e renda per capita mensal, Ribeirão Preto, 2009.

\begin{tabular}{ccccccc}
\hline Classificação & \multicolumn{2}{c}{ RPIM } & \multicolumn{2}{c}{ RPSM } & \multicolumn{2}{c}{ Total } \\
\cline { 2 - 7 } & $\mathbf{n}$ & $\mathbf{\%}$ & $\mathbf{n}$ & $\mathbf{\%}$ & $\mathbf{n}$ & $\mathbf{\%}$ \\
\hline I.A. Grave & 7 & 100,00 & - & - & 7 & 100,00 \\
I.A. Moderada & 17 & 65,38 & 9 & 34,62 & 26 & 100,00 \\
I.A. Leve & 4 & 33,33 & 8 & 66,67 & 12 & 100,00 \\
S.A. & 3 & 20,00 & 12 & 80,00 & 15 & 100,00 \\
\hline Total & 31 & 51,67 & 29 & 48,33 & 60 & 100,00 \\
\hline
\end{tabular}

$\mathrm{p}=0,001$

Fonte: Dados de pesquisa. 
A média de número de refeições realizadas pelos alunos foi de cinco - duas delas realizadas por meio da alimentação escolar. Apenas $15 \%$ deles $(n=9)$ podiam contar com mais duas refeições oferecidas pelo núcleo de atendimento da criança e do adolescente mantido pela prefeitura.

O equivalente a $58,33 \%$ das crianças $(n=35)$ relataram consumir a merenda escolar diariamente, enquanto $30 \%$ dos alunos $(n=18)$ revelaram consumir a merenda apenas quando eram oferecidos alimentos de seu gosto, entre os quais foram citados principalmente frango empanado frito e macarrão.

Conforme a Tabela 2, o consumo da merenda escolar foi significativamente maior entre as crianças pertencentes a famílias com renda per capita inferior à mediana.

Tabela 2 - Distribuição dos alunos participantes da pesquisa $(n=60)$ de acordo com o consumo da merenda escolar e renda per capita mensal, Ribeirão Preto, 2009.

\begin{tabular}{ccccccc}
\hline Consumo da & \multicolumn{2}{c}{ RPIM } & \multicolumn{2}{c}{ RPSM } & \multicolumn{2}{c}{ Total } \\
\cline { 2 - 7 } & $\mathbf{n}$ & $\mathbf{\%}$ & $\mathbf{n}$ & $\mathbf{\%}$ & $\mathbf{n}$ & $\mathbf{\%}$ \\
\hline Sim & 23 & 65,71 & 12 & 34,29 & 35 & 100,00 \\
Não & 2 & 28,57 & 5 & 71,43 & 7 & 100,00 \\
Às vezes & 6 & 33,33 & 12 & 66,67 & 18 & 100,00 \\
\hline Total & 31 & 51,67 & 29 & 48,33 & 60 & 100,00 \\
\hline
\end{tabular}

$\mathrm{p}<0,05$

Fonte: Dados de pesquisa.

Quanto à satisfação dos alunos com relação à merenda escolar, segundo relato da mãe ou do responsável pela criança, $71,67 \%$ dos pesquisados $(n=43)$ revelaram que a criança gosta da merenda servida e apenas $13,33 \%(n=8)$ informaram que a criança não gosta da refeição servida.

Um estudo realizado no município de Piracicaba observou que $77,5 \%$ dos alunos entrevistados afirmaram realizar as refeições servidas na escola, e a maioria consumia pelo fato de gostar da alimentação, além do relato de outros fatos como: sentir fome, por não almoçar em casa, por considerar a merenda melhor que a alimentação da casa ou devido ao fato de não ter dinheiro para comprar alimentos na cantina
(MARTINS et al., 2004).

No presente estudo, apenas 63,33\% $(n=38)$ dos pais ou responsáveis pela criança tinham conhecimento sobre o tipo de refeição servida na escola; desses, $71,05 \%(n=27)$ a classificaram como boa e $28,95 \%$ ( $n=11)$, como ótima, e nenhum entrevistado classificou a refeição servida na escola como ruim.

O Quadro 1 mostra o número e porcentagens de crianças quanto à realização das refeições durante todo o dia; $75 \%(n=45)$ almoçam em casa e também na escola, sendo esta última refeição chamada de almoço 2. Do total de crianças estudadas, $30 \%(n=18)$ realiza outras refeições, como, por exemplo, um segundo lanche da tarde. 


\begin{tabular}{|l|c|c|}
\hline \multicolumn{1}{|c|}{ Refeições } & n & \% \\
\hline Café da manhã & 50 & 83,33 \\
\hline Colação & 13 & 21,67 \\
\hline Almoço 1 & 54 & 90,00 \\
\hline Almoço 2 & 45 & 75,00 \\
\hline Lanche da Tarde & 52 & 86,67 \\
\hline Jantar & 59 & 98,33 \\
\hline Ceia & 27 & 45,00 \\
\hline Outras & 18 & 30,00 \\
\hline
\end{tabular}

Quadro 1 - Distribuição das refeições consumidas diariamente pelos alunos com relação ao total da amostra.

Fonte: Dados de pesquisa.

De acordo com Sturion et al. (2005), em um estudo realizado para verificar o nível de adesão dos alunos ao Programa Nacional de Alimentação Escolar, a frequência de consumo da alimentação escolar foi maior entre os alunos que recebem até três refeições no domicílio e menor entre os que recebem mais de três refeições no domicílio. Provavelmente, os escolares que consumiam menor número de refeições no domicílio eram de classes de menor renda familiar e, portanto, consumiam a alimentação escolar.

Quase $84 \%$ das crianças tomam o café da manhã; este dado é bastante importante, uma vez que essa refeição é bastante importante para os indivíduos, em especial para as crianças; Kleinman et al. (1998), a partir de revisões dos efeitos do café da manhã na cognição e desempenho escolar, sugerem que crianças que vão para a escola sem o café da manhã terão deficiências em seu desempenho escolar maior do que as que realizam a refeição.

Houve diferença extremamente significativa $(p<0,001)$ entre os grupos de renda em relação à mediana quanto à realização da ceia, refeição esta realizada por 27 crianças, das quais 21 (77,78\%) faziam parte do grupo de RPSM. O dado indica que a realização da ceiaé mais comum entre as crianças com renda mais elevada. Em contrapartida, se a mesma comparação for feita entre crianças em situação de segurança e insegurança alimentar, pode-se verificar que a realização da ceia é mais comum entre os indivíduos em situação de insegurança alimentar, com $62,96 \%$ dos indivíduos $(\mathrm{p}=0,05)$. 


\begin{tabular}{|l|c|c|c|}
\hline \multicolumn{1}{|c|}{ Grupo dos Alimentos } & Média do & Mínimo & Máximo \\
& número de & Recomendado & Recomendado \\
\hline Pães, cereais, raízes e tubérculos & & 5 & 9 \\
\hline Hortaliças & 2 & 4 & 5 \\
\hline Frutas & 1 & 3 & 5 \\
\hline Carnes & 3 & 1 & 3 \\
\hline Leite & 2 & 3 & 1 \\
\hline Leguminosas: & 2 & 1 & 2 \\
\hline Óleos e gorduras: & 1 & 1 & 2 \\
\hline Açúcares e doces & 1 & 1 & \\
\hline
\end{tabular}

Quadro 2 - Distribuição dos grupos dos alimentos quanto à média do número de porções consumidas pelos alunos, comparadas com o número mínimo e o máximo de porções recomendado pela pirâmide alimentar adaptada.

Fonte: Dados de pesquisa.

Conforme o Quadro 2, quanto ao consumo alimentar, houve uma adequação da média de ingestão de cinco porções do grupo 1 (pães, cereais, raízes e tubérculos), uma vez que a pirâmide alimentar preconiza um consumo entre cinco e nove porções ao dia. O mesmo ocorreu com o grupo dos óleos e gorduras e o dos açúcares e doces, cujo consumo deve ser de uma a duas porções diárias para cada um desses grupos, e foi exatamente o valor mínimo relatado sobre o consumo desses tipos de alimentos.

Houve umexcesso na ingestão do grupo das carnes, com três porções ao dia, contrariamente ao consumo preconizado de, no máximo, duas porções diárias. Isso ocorre porque um grande número de crianças $(n=35)$, representando $58 \%$ da amostra, realizava as duas grandes refeições em casa e, além disso, consumiam a refeição servida na escola, cujo cardápio, na maioria das vezes, é composto por uma refeição completa, incluindo basicamente arroz, feijão, um tipo de carne e uma hortaliça. Pelo mesmo motivo, o consumo de três porções de feijão ao dia também se mostrou elevado, uma vez que a pirâmide alimentar indica um consumo máximo de uma porção ao dia. Em contrapartida, no que diz respeito aos grupos das hortaliças, das frutas e do leite, o consumo de duas, uma e duas porções, respectivamente, foi aquém das recomendações mínimas estabelecidas pela pirâmide - de quatro porções de hortaliças e três porções de frutas e também de leite.

É de suma importância que frutas e hortaliças façam parte da dieta cotidiana da população, substituindo alimentos de alta densidade energética e com menor teor de nutrientes e de fibras, levando em consideração o cenário epidemiológico de intenso crescimento da obesidade e de doenças crônicas associadas à dieta no Brasil e em todo mundo (CLARO et al., 2007).

Considerando que a merenda é uma importante fonte de acesso da criança aos alimentos, este estudo também se propôs a analisar a merenda escolar, a fim de verificar a qualidade nutricional desta com base no valor energético oferecido, bem como a sua distribuição em macro e micronutrientes.

A legislação em vigor referente à alimentação escolar na data da pesquisa era a Resolução n. ${ }^{\circ}$ 32, de 10 de agosto de 2006 (BRASIL, 2006), que preconizava um aporte energético de 300 quilocalorias com 4,2 gramas de proteína. Dessa forma, a alimentação servida pôde ser considerada de alto valor calórico e proteico, 
uma vez que apresentou valores bastante superiores ao estipulado para esses critérios.

Porém, se considerarmos a resolução atualmente em vigor (BRASIL, 2009), podemos verificar, no Quadro 3, uma melhor adequação da dieta, até porque esta preconiza valores energéticos e de nutrientes mais adequados, uma vez que considera a idade do aluno, bem como o número de refeições servidas pela escola, com valores maiores quanto à energia e proteína e os valores médios ideais para micronutrientes.

\begin{tabular}{|l|c|c|c|}
\hline & PNAE (2009) & Oferecido & Adequação (\%) \\
\hline Valor Energético (kcal) & 450 & 491,01 & 109,11 \\
\hline Carboidratos (g) & 73,1 & 70,69 & 96,70 \\
\hline Proteínas (g) & 14,0 & 21,44 & 153,14 \\
\hline Lipídeos (g) & 11,3 & 13,61 & 120,44 \\
\hline Fibras $(\mathrm{g})$ & 8,0 & 2,937 & 36,71 \\
\hline Vitamina A ( $\mu \mathrm{g})$ & 150,0 & 85,528 & 57,02 \\
\hline Vitamina C (mg) & 11,0 & 9,846 & 89,51 \\
\hline Cálcio $(\mathrm{mg})$ & 315,0 & 295,021 & 93,66 \\
\hline Ferro $(\mathrm{mg})$ & 2,7 & 2,448 & 90,67 \\
\hline Magnésio $(\mathrm{mg})$ & 56,0 & 68,784 & 122,83 \\
\hline Zinco $(\mathrm{mg})$ & 2,0 & 1,535 & 76,75 \\
\hline
\end{tabular}

Quadro 3 - Comparação entre os valores de referência preconizados pelo Programa Nacional de Alimentação Escolar (2009) e os valores médios oferecidos pela escola estudada.

Fonte: Dados de pesquisa.

Adotando-se uma porcentagem de adequação ideal entre 90 e $110 \%$, podemos dizer que a alimentação escolar oferecida pela instituição estudada, de acordo com a atual resolução, está adequada quanto ao valor energético, de carboidratos, de cálcio e de ferro. As quantidades de proteína, lipídeo e magnésio oferecidas são bastante superiores com relação ao que é preconizado pelo programa; e quanto às fibras, vitamina A, vitamina $\mathrm{C}$ e zinco, os valores oferecidos estão aquém das necessidades nutricionais dos alunos, de acordo com a Resolução n. ${ }^{\circ} 38$ do Fundo Nacional de Desenvolvimento da Educação, a qual estabelece os valores ideais a serem oferecidos para a alimentação escolar de crianças entre 6 e 10 anos.

\section{Conclusão}

Conclui-se que a população estudada é composta, em sua maioria, por famílias de baixa condição socioeconômica e em situação de insegurança alimentar moderada.

A maioria das crianças (65\%) encontra-se com estado nutricional adequado, de acordo com a avaliação antropométrica; entretanto, é considerável o número de crianças com excesso de peso $(26,67 \%)$.

Houve significantes inadequações quanto ao consumo alimentar, o que requer uma intervenção voltada para educação alimentar e nutricional, uma vez que o consumo de hortaliças, frutas e leite foi baixo, indicando um possível estado nutricional inadequado no que diz respeito a vitaminas e minerais, importantes para o crescimento, desenvolvimento e manutenção das funções do organismo de qualquer indivíduo, em especial das crianças.

A condição socioeconômica mostrou ter influência 
no estado nutricional dos escolares estudados, uma vez que a renda e a condição de segurança alimentar foram determinantes para que a criança dispusesse de melhor acesso aos alimentos, apresentando assim um consumo mais adequado do ponto de vista nutricional, com base nos mínimos estipulados para uma alimentação saudável.

A situação de insegurança alimentar esteve mais presente em famílias de baixa renda, apesar de ser diagnosticada também em famílias com renda superior à linha da pobreza, e do fato de famílias pobres estarem em situação de segurança alimentar.

A alimentação escolar se mostrou de grande importância para as crianças, principalmente as de condição socioeconômica menos favorecida; foi considerada satisfatória quanto a calorias, carboidratos, cálcio e ferro, embora tenha apresentado valores superiores quanto a proteínas e lipídeos. A atual resolução pareceu mais adequada, pois permite um valor calórico e proteico maior, uma vez que $40 \%$ do total de refeições realizadas pelas crianças ocorrem por meio da alimentação escolar.

\section{CONSIDERAÇõES FINAIS}

Apesar da demonstração exaustiva da associação positiva da condição socioeconômica sobre a situação de segurança alimentar e, consequentemente, no estado nutricional dos indivíduos, é importante enfatizar a influência desta nos hábitos alimentares de crianças em idade escolar, considerando inclusive que variáveis como a renda e a escolaridade dos pais são importantes fatores condicionantes para escolhas alimentares, pois determinam a compra de acordo com a quantidade de dinheiro disponível e o fato de possuírem mais informações e conhecimento sobre alimentação e saúde.

Os dados deste estudo reforçam essa relação, uma vez que a análise da ingestão de nutrientes é uma maneira para detecção de inadequações quanto à alimentação e, consequentemente, um indicativo do estado de nutrição ou subnutrição do indivíduo; além disso, reforça também a importância da merenda escolar e de programas sociais que visem ao suprimento alimentar adequado a crianças e adolescentes.

A alimentação escolar é uma complementação da alimentação que a criança deve receber em casa e, portanto, é importante que o restante dos alimentos consumidos pela criança, fora do horário letivo, esteja em adequação, a fim de evitar que a mesma apresente carências nutricionais e/ou problemas relativos ao consumo excessivo de determinados nutrientes.

$\mathrm{O}$ acesso à alimentação adequada é um direito de todo e qualquer cidadão e deve ser garantido, incluindo medidas de curto, médio e longo prazos. Programas de transferência de renda são uma maneira de amenizar o problema da situação de insegurança alimentar e da desigualdade social em curto prazo, uma vez que grande parte das famílias entrevistadas conta com esse benefício como única renda fixa do domicílio. Entretanto, é indispensável a adoção de medidas que visem a resultados em médio e longo prazos, com a implantação de frentes de trabalho para empregar as pessoas e as tirarem da condição de pobreza, proporcionando dignidade e melhores condições de vida para a família.

As precárias condições de moradia são ainda um grande problema de infraestrutura e de saúde pública, tendo em vista que as condições de higiene do local podem prejudicar a qualidade de vida e a saúde dos moradores, principalmente das crianças.

Porém, o resultado de todas essas mudanças propostas seria apenas parcial ou até mesmo todas essas medidas seriam ineficazes se, junto a elas, não for estabelecido um programa educacional que vise à adoção de bons hábitos alimentares, com a adequada utilização financeira da renda e até mesmo dos benefícios dos programas sociais.

Para que seja feita uma intervenção eficaz, que proporcione custo-benefício favorável, todos os parâmetros relacionados com o estado nutricional dos indivíduos devem ser considerados, até porque somente o peso e a estatura não revelam, de modo completo, o real estado nutricional de um indivíduo.

A partir de uma investigação mais detalhada, como, por exemplo, com uma avaliação bioquímica, seria possível detectar patologias nutricionais e/ou condições 
de risco, e consequentemente diagnosticar crianças em estado nutricional inadequado. Entretanto, o fato de o consumo alimentar não ter se mostrado adequado quanto a alguns grupos alimentares, já é um indicativo de um estado pré-patológico de carências nutricionais, permitindo indagar se as crianças eutróficas que estão em situação de insegurança alimentar realmente apresentam um bom estado de saúde, enfatizando a importância da educação nutricional para a melhoria da qualidade de vida da população.

Este estudo não tem a intenção de esgotar o assunto, uma vez que são necessárias ainda muitas pesquisas que tragam avanços com relação à segurança alimentar da população, mas trata-se de uma junção de diversas variáveis, que merecem atenção na investigação do tema.

\section{REFERÊNCIAS}

BEGHIN, I. Abordagem Causal em Nutrição. In: GOUVEIA, E.L.C. (Org.). Nutrição, Saúde e Comunidade. 2. ed. Rio de Janeiro: Revinter, 1999. cap. 7, p. 179-190.

BRASIL. Ministério da Educação e Cultura. Fundo Nacional de Desenvolvimento da Educação.

Resolução/CD/FNDE N. ${ }^{\circ}$ 38, de 16 de Julho de 2009. Brasília: MEC, 2009.

- Ministério da Educação e Cultura. Fundo Nacional de Desenvolvimento da Educação.

Resolução/CD/FNDE N. ${ }^{\circ}$ 32, de 10 de agosto de 2006. Brasília: MEC, 2006 b.

Ministério da Saúde. Secretaria de

Atenção à Saúde. Departamento de Atenção Básica. Coordenação Geral da Política de Alimentação e Nutrição. Vigilância Alimentare Nutricional SISVAN: Orientações para a coleta e análise de dados antropométricos em serviços de saúde. Brasília: Ministério da Saúde, 2008. 7p. . Ministério da Saúde. Vigilância
Alimentar e Nutricional - SISVAN: Orientações básicas para a coleta, processamento, análise de dados e informação em serviços de saúde. Brasília: Ministério da Saúde, 2004a. 120p.

. Ministério do Planejamento, Orçamento e Gestão. Instituto Brasileiro de Geografia e Estatística. Pesquisa Nacional por Amostra de Domicílios: Questionário da Pesquisa de 2004. Rio de Janeiro: Ministério do Planejamento, Orçamento e Gestão, 2004b. 61p.

Ministério do Planejamento, Orçamento e Gestão. Instituto Brasileiro de Geografia e

Estatísticas. Indicadores Sociais Municipais. Rio de Janeiro: Ministério do Planejamento, Orçamento e Gestão, 2004c. 62p.

Ministério do Planejamento, Orçamento e Gestão. Instituto Brasileiro de Geografia e Estatísticas. Síntese de Indicadores Sociais. Rio de Janeiro: Ministério do Planejamento, Orçamento e Gestão, 2006b. 317p.

BURLANDY, L. Transferência condicionada de renda e segurança alimentar e nutricional. Ciênc. Saúde Coletiva, v. 12, n. 6, p. 1441-1451, nov./ dez. 2007.

BURLANDY, L.; ANJOS, L.A. dos. Acesso à alimentação escolar e estado nutricional de escolares no Nordeste e Sudeste do Brasil, 1997. Cad.

Saúde Pública, Rio de Janeiro, v. 23, n. 5, p. 1217 1226, mai. 2007.

CLARO, R.M.; CARMO, H.C.E.; MACHADO, F.M.S.; MONTEIRO, C.A. Renda, preço dos alimentos e participação de frutas e hortaliças na dieta. Rev. Saúde Pública, v. 41, n. 4, p. 557564, 2007.

DOMENE, S.M.A.; OTA, R.R.L.; NILSON, E. A. F.; OLLERTZ, M.I.S.; WATANABE, T.T.; 
GALLO, P.R. Experiências de políticas em alimentação e nutrição. Estud. Av., v. 21, n. 60, p. 161-178, mai./ago. 2007.

FERREIRA, H.S. O estado nutricional da população brasileira. In:

Desnutrição: Magnitude, significado social e possibilidade de prevenção. Maceió: EDUFAL, 2000. cap. 1, p. 25-30.

KLEINMAN, R.E.; MURPHY, J.M.; LITTLE, M. et al. Hunger in children in the United States: potencial behavioral and emotional correlates. Pediatrics. v. 101, n. 1, p.1-6, jan. 1998.

LIBERONA, Y.; ENGLER, V.; CASTILLO, O.; VILLARROEL, L.; ROZOWSKI, J. Ingesta de macronutrientes y prevalencia de malnutrición por exceso en escolares de $5^{\circ}$ y $6^{\circ}$ básico de distinto nivel socioeconómico de la región metropolitana. Rev. Chil. Nutr., v. 35, n. 3, p. 190-199, sep. 2008.

LIMA, M.C.; GOUVEIA, E.L.C. Avaliação nutricional em coletividades. In: GOUVEIA, E.L.C. (Org.). Nutrição, Saúde e Comunidade. 2. ed. Rio de Janeiro: Revinter, 1999. cap. 6, p. 137-177.

MARTINS, R.C.B.; MEDEIROS, M.A.T.; RAGONHA, G.M.; OLBI, J.H.; SEGATTI, M.E.P.; OSELE, M.R. Aceitabilidade da Alimentação Escolar no Ensino Público Fundamental. Saúde Rev., v. 6, n. 13, p. 71-78, jul. 2004.

MONTEIRO, C.A.; BENICIO, M.H.A.; KONNO; S.C.; SILVA; A.C.F.; LIMA; A.L.L.; CONDE; W.L. Causas do declínio da desnutrição infantil no Brasil, 1996-2007. Rev. Saúde Pública, v. 43, n. 1, p. 35-43, 2009.

PHILIPPI, S.T. Guia Alimentar para o Ano 2000. In: ANGELIS, R.C. (Org.). Fome Oculta: Bases Fisiológicas para reduzir seus riscos através da alimentação saudável. São Paulo: Atheneu, 2000. cap. 32, p. 160-176.

\section{PHILIPPI, S.T. Tabela de Composição de}

Alimentos: Suporte para Decisão Nutricional. 2.ed., São Paulo: Produção Independente, 2002. $130 \mathrm{p}$.

PHILIPPI, S.T.; LATTERZA, A.R.; CRUZ, A.T.R.; RIBEIRO, L.C. Pirâmide alimentar adaptada: guia para escolha dos alimentos. Rev. Nutr., Campinas, v. 12, n. 1, p. 65-80. jan./abr. 1999.

SANTOS, R.B.; MARTINS, P.A.; SAWAYA, A.L. Estado nutricional, condições socioeconômicas, ambientais e de saúde de crianças moradoras em cortiços e favela. Rev. Nutr., Campinas, v. 21, n. 6, p. 671-681, nov./dez. 2008.

SEGALL-CORREA, A.M. Insegurança alimentar medida a partir da percepção das pessoas. Estud. Av., v. 21, n. 60, p. 143-154, 2007.

STURION, G.L.; SILVA, M.V.; OMETTO, A.M.H.; FURTUOSO, M.C.O.; PIPITONE, M.A.P. Fatores condicionantes da adesão dos alunos ao Programa de Alimentação Escolar no Brasil. Rev. Nutr., Campinas, v. 18, n. 2, p. 167 181, mar./abr. 2005.

TARTAGLIA, J.C. Industrialização, Alimentação e Segurança Alimentar no Brasil. In: DUTRA-DEOLIVEIRA, J.E.; MARCHINI, J.S. (Orgs.). Ciências Nutricionais. São Paulo: Sarvier, 1998. cap. 20, p. 323-351.

TIRAPEGUI, J.; MENDES, R.R. Introdução à Nutrição. In: TIRAPEGUI, J. (Org.). Nutrição: Fundamentos e Aspectos Atuais. 2.ed. São Paulo: Atheneu, 2006. cap. 1, p. 1-5.

VIANNA, R.P.T.; SEGALL-CORREA, A.M. Insegurança alimentar das famílias residentes em 
municípios do interior do estado da Paraíba, Brasil. Rev. Nutr., Campinas, v.21, suppl., p. 111-122, jul./ago. 2008.

VITOLO, M.R. Práticas Alimentares na Infância. In: Nutrição: da Gestação ao Envelhecimento. Rio de Janeiro: Ed. Rubio, 2008. cap. 23, p. 215-242.

ZANCUL, M.S. Consumo alimentar de alunos nas escolas de ensino fundamental em Ribeirão Preto (SP). 2004. 85f. (Dissertação) Faculdade de Medicina de Ribeirão Preto, Ribeirão Preto, 2004. 